\title{
Leukocyte telomere shortening in Huntington's disease
}

${ }^{\#}$ Daniela Scarabino ${ }^{\mathrm{a}}{ }^{\#}$ Liana Veneziano $^{\mathrm{b}}$, Martina Peconi ${ }^{\mathrm{b}, \mathrm{c}}$, Marina Frontali ${ }^{\mathrm{b}}$, E. Mantuano ${ }^{\mathrm{b}}$, R.M. $\mathrm{Corbo}^{\mathrm{c}, \mathrm{a} *}$.

\# These authors contributed equally to this work

${ }^{\text {a }}$ CNR Institute of Molecular Biology and Pathology, c/o Department of Biology and Biotechnology, P.le Aldo Moro 5, 00185 Rome, Italy.

${ }^{\mathrm{b}}$ CNR Institute of Translational Pharmacology, Via Fosso del Cavaliere 100, 00133 Rome, Italy

${ }^{\mathrm{c}}$ Department of Biology and Biotechnology, La Sapienza University, P.le Aldo Moro 5, 00185 Rome, Italy

*Corresponding Author: Prof. Rosa Maria Corbo,

Department of Biology and Biotechnology “Charles Darwin”, La Sapienza University

P.le Aldo Moro 5, 00185, Rome, Italy.

Tel. ++39.6 .49912825$

e-mail: rosamaria.corbo@uniroma1.it

Short title: Telomere length in Huntington's disease.

Key words: Neurodegenerative disease, Huntington's disease development, Leukocyte telomere length, biomarker 


\section{Abstract}

Huntington's disease (HD) is an autosomal dominant neurodegenerative disease caused by an expanded CAG repeat. Though symptom onset commonly occurs at midlife and inversely correlates with the CAG repeat expansion, age at clinical onset and progression rate are variable. In the present study we investigated the relationship between leukocyte telomere length (LTL) and HD development. LTL was measured by real-time PCR in manifest HD patients (HD, $n=62$ ), pre-manifest HD patients (pre-HD, $n=38$ ), and age-matched controls ( $n=$ 76). Significant LTL differences were observed between the three groups ( $p<0.0001)$, with LTL values in the order: HD < pre-HD <controls. The relationship between LTL and age was different in the three groups. An inverse relationship between mean LTL and CAG repeat number was found in the pre-HD ( $\mathrm{p}=0.03)$. The overall data seem to indicate that after age 30 years, LT begins to shorten markedly in pre-HD patients according to CAG number and increasing age, up to the values observed in HD. This very suggestive picture allowed us to hypothesize that in pre-manifest HD, LTL could be a measure of time to clinical HD onset. The possible use of LTL as a reliable biomarker to track HD development and progression was evaluated and discussed. 


\section{Introduction}

Huntington's disease (HD) is an autosomal dominant, progressive neurodegenerative disorder caused by a CAG trinucleotide repeat expansion in the first exon of the HTT gene encoding huntingtin (HTT). The mutant protein contains an expanded polyglutamine sequence (poly-Q) that confers a toxic gain of function and results in neurodegeneration. The disease is fully penetrant in individuals with $\geq 40$ repeats, with onset of motor symptoms in mid-age, often in the fourth or fifth decade. Overall the age of onset is inversely correlated with the size of the CAG repeat expansion, but actually the age of clinical onset, rate of disease progression, and severity of symptoms vary widely among individuals. CAG repeat length accounts for about $56 \%$ of the variation in age at clinical onset within a range of 40 to 55 CAG repeats. Since the remaining variance in age at motor onset is not explained by the length of the CAG repeat, it cannot be used to accurately predict when symptoms will occur. This residual variance is probably due to genetic, stochastic, and environmental factors [1-3]. The challenge is to identify objectively measurable peripheral biomarkers related to CAG repeat length in a statistical tool to predict age of onset and disease progression.

Human telomeres consist of repeated TTAGGG nucleotide sequences located at the ends of chromosomes where they protect them against from DNA damage. During DNA replication, telomeres shorten progressively with repeated cell divisions due to the inability of DNA polymerase to replicate the $3^{\prime}$ end of the DNA strand. A cellular multi-protein complex, called telomerase, counteracts telomere shortening $[4,5]$. Usually present in the early stages of embryonic development, its activity is silenced in several human somatic tissues immediately after birth [6]. As a consequence, in the telomeres shorten progressively in the replicating cells of adult tissues (including skin, kidney, liver, blood vessels, and peripheral leukocytes); this phenomenon is thought to indicate cellular age and reflect an organism's biological age [7-9].

By virtue of tissue availability, measurement of leukocyte telomere length (LTL) is widely used as a marker of overall telomere length assuming that, within a given individual, the TL of various tissue types is strongly correlated [10]. Epidemiological studies have provided evidence for the hypothesis that leukocyte telomere shortening is associated with aging 
$[7,8,9,11]$ and with age-related chronic diseases (cardiovascular and metabolic diseases, cancer), although some inconsistencies have been observed [11-13]. LTL was also investigated in connection with such neurodegenerative diseases as Alzheimer's disease (AD), Parkinson's disease (PD), and HD. Despite some discordant results, shorter leukocyte telomeres were frequently found in association with cognitive decline/dementia, $\mathrm{AD}$ and its prodromal state, MCI (mild cognitive impairment) [14-16]. Recently, we were able to identify a significant progressive leukocyte telomere reduction in patients with $\mathrm{MCI}$ and $\mathrm{AD}$ patients as compared to controls [17]. This finding suggests that LTL measurement could provide a useful marker to follow dementia progression from its prodromal stages (MCI) to manifest AD. Studies on telomere length in PD patients showed no significant evidence of shorter telomere length [18]. In contrast, in the only study carried out on HD to our knowledge, significantly shorter LTL was observed in manifest HD patients as compared to controls [19].

$\mathrm{AD}$ and HD share several common features, including neuronal loss, accumulation of aggregated and misfolded proteins, and cognitive decline. On account of this, we thought it interesting to determine whether LTL is reduced in manifest HD patients and to investigate LTL in pre-manifest $\mathrm{HD}$, i.e., at the disease stage in which, as observed in the AD prodromal stage (MCI), telomere shortening presumably begins and progresses to reach LTL values observed in manifest HD. If so, the telomere measurement could be a useful parameter to follow the development of the disease. Unlike persons with MCI in whom the course of the disease towards $\mathrm{AD}$ is uncertain, $\mathrm{HD}$ patients with $\mathrm{CAG}$ repeat expansion in the pre-manifest stage will inexorably develop the disease, although the age at disease onset is highly variable. Indeed, the inverse relationship between $\mathrm{HD}$ age at onset and the number of CAG repeats, is stronger for the alleles with 60 repeats or higher but weaker for the most frequent HD alleles (55 or fewer) [1]. Some models have been developed to estimate the years until onset in mutation carriers on the basis of age and CAG repeat length [20], but their usefulness may be limited by the complexity of the HD clinical phenotype, to whose manifestation other genetic and environmental factors may take part [21]. In this context, the search for reliable biological 
markers that can contribute to predict age of onset and disease progression is crucial in both genetic counseling and trials of disease-modifying drugs that could delay disease onset.

Here, we analyzed LTL in a sample of pre-manifest (pre-HD) and manifest HD (HD) patients. We then compared LTL with relevant disease parameters (number of repeats, age at disease onset, disease duration) to gain a clearer picture of the relationships between telomere length and HD development and progression. Measurement of LTL in HD mutation carriers at different stages of the disease (pre-manifest and manifest with different disease duration) could prove to be a useful non-invasive tool to predict clinical onset in pre-manifest HD patients and disease progression in manifest HD patients.

\section{Materials and Methods}

\subsection{Subjects}

The study population consisted of 62 unrelated patients with clinical diagnosis of HD, 38 pre-manifest mutation carriers, and 76 age-matched control subjects collected over years at the Institute of Translational Pharmacology, National Research Council, referred from various neurological centres of Rome (Italy). Diagnosis was performed by an experienced HD neurologist on the basis of the Unified Huntington's Disease Rating Scale (UHDRS) using a Total Motor Score $\geq 4$, for the diagnosis of HD [22]. Among HD patients about 50\% were at an early disease stage, and $50 \%$ at a moderate disease stage [22].

Clinical data are reported in Table 1. Age considered for the analysis was the age at the time of blood collection. Age at onset was available only for 40 patients and was estimated as the age at which motor and/or cognitive symptoms first occurred. Control subjects were recruited from among patients' healthy relatives and spouses, who were suitable to be matched for age and gender and agreed to participate in the study. Written, informed consent was obtained from all participants. The protocol for the collection of DNAs for research purposes was approved by the institutional ethics committees according to the principles expressed in the Declaration of Helsinki.

\subsection{Laboratory Methods}


Venous blood was drawn in EDTA from all subjects after overnight fasting. Genomic DNA was extracted from whole blood using the salting out method described by Miller et al. [23]. HD molecular diagnosis was performed in duplicate by PCR amplification with primers HD1/HD3 [24]; DNA fragments were analyzed by capillary electrophoresis. The results were confirmed by triplet repeat primed PCR (TP PCR) [25].

The average (of triplicate) telomere length in leukocytes was measured by monoplex real-time PCR quantitative analysis (monoplex qPCR) on a 7300 real-time PCR instrument (Applied Biosystems). This method allows the determination of the number of copies of telomeric repeats $(\mathrm{T})$ compared to a single copy gene (S) used as a quantitative control (T/S ratio) [26]. The telomere and single-copy gene $\beta$-globin (HGB) were analyzed on the same plate in order to reduce inter-assay variability. DNA (35 ng) was amplified in a total volume of $20 \mu \mathrm{l}$ containing $10 \mu \mathrm{l}$ of SYBER Select Master Mix (Applied Biosystems); primers for telomeres and the single-copy gene were added to final concentrations of $0.1 \mu \mathrm{M}$ (Tel Fw), $0.9 \mu \mathrm{M}$ (Tel Rev) and $0.3 \mu \mathrm{M}$ (HGB Fw), $0.7 \mu \mathrm{M}$ (HGB Rev), respectively. The primer sequences were: Tel Fw 5'-CGGTTTGTTTGGGTTTGGGTTTGGGTTTGGGTTTGGGTT-3'; Tel Rev 5'GGCTTGCCTTACCCTTACCCTTACCCTTACCCTTACCCT-3'; HGB Fw 5'GCTTCTGACACAACTGTGTTCACTAGCAAC-3'; HGB Rev 5'CACCACCAACTTCATCCACGTTCACCTTGC-3' [26]. The enzyme was activated at $95^{\circ} \mathrm{C}$ for $10 \mathrm{~min}$, followed by 40 cycles at $95^{\circ} \mathrm{C}$ for $15 \mathrm{~s}$ and $60^{\circ} \mathrm{C}$ for $1 \mathrm{~min}$. In addition, two standard curves (one for HGB and one for telomere reactions), were prepared for each plate using a reference DNA sample (Control Genomic Human DNA, Applied Biosystems) diluted in series (dilution factor of 2) to produce 5 concentrations of DNA ranging from 50 to $6.25 \mathrm{ng}$ in $20 \mu \mathrm{L}$. Measurements were performed in triplicate and are reported as $\mathrm{T} / \mathrm{S}$ ratio relative to the calibrator sample to allow comparison across runs. Replicate assays of same sample were carried out to calculate the interassay variation. The average standard deviation as calculated by measuring relative $\mathrm{T} / \mathrm{S}$ ratios of a sample repeated over three different assays was $4.2 \%$. Thus, assuming a normal distribution, samples differing in average telomere length by as little as $8.3 \%$ (1.96 X SD) should be distinguishable by this method at the $95 \%$ confidence interval [26]. No 
amplification of the negative controls with both primer sets (HGB and telomeres) used was observed.

\subsection{Statistical analysis}

Parametric (ANOVA) and nonparametric (Kruskal-Wallis) tests were used to compare the distribution of quantitative variables between pre-manifest and manifest HD patients and controls, and the distribution of the mean T/S ratio across age and repeat number classes. Level of significance was set at $\mathrm{p}<0.05$. The relationship between $\mathrm{T} / \mathrm{S}$ ratio and age was evaluated by regression analysis. LTL was adjusted for age by regression for further analyses. Comparison of the regression lines slopes was carried out by a t-test.

The estimated age at onset was calculated according to the formula of Langbehn et al. [20], at different probability values, given CAG repeat number and age at blood sampling (see http://edwild.com/205-huntingtons-disease-conditional-onset-probability-calculator/). The time to clinical diagnosis was calculated as the difference between estimated onset and age at blood sampling.

\section{Results}

Leukocyte telomere length (LTL), expressed as T/S ratio, was measured in controls, pre-manifest HD, and manifest HD patients. Table 1 presents the demographic and clinical characteristics of the three groups. The difference in mean age between the groups $(p<0.001)$ depended on the disease stage (pre-manifest and manifest) of HD patients, while the age range of the control sample corresponded to that of the HD patients (both pre-manifest and manifest). A statistically significant difference in the LTL distribution between the control group and the two patient groups (Fig. 1) was observed using Kruskal-Wallis test ( $\mathrm{p}<0.00001$ ). The LTL values showed a decreasing trend in the three groups, in the order control LTL > pre-manifest HD LTL >manifest HD. Post hoc pair-wise comparison showed that LTL values differed significantly from one another in the three groups (Fig. 1). No difference in mean LTL was observed between males and females in the three groups (controls: $\mathrm{p}=0.11$, pre-manifest HD: $\mathrm{p}=0.99$, manifest HD patients: $\mathrm{p}=0.54)$. 
Linear regression analysis showed a significant negative relationship between LTL and age for each group (controls: $y=-0.0027 x+1.06, p<0.0001$; pre-manifest HD: $y=-0.007 x+$ $1.05, p=0.0005 ; \mathrm{HD}: \mathrm{y}=-0.002 \mathrm{x}+0.70, \mathrm{p}=0.004)$. Comparison of the regression lines slopes by a t-test showed that the decreasing trend for telomere length with age differed significantly between the pre-manifest HD group as compared with the controls $(\mathrm{p}=0.03)$ and with the HD group ( $\mathrm{p}=0.02)$. This was confirmed when we compared the LTL values for the different age classes of the three groups (Table 2). There was an apparent downward trend of LTL with increasing age for the controls, which began to grow at the more advanced ages (Table 2). In pre-manifest $\mathrm{HD}$, the distribution by age class was uneven, with fewer patients of older age, as expected. The LTL of the very young pre-manifest HD patients (age class 20-29, LTL=0.91) was very similar to that reported in the age-matched controls $(\mathrm{LTL}=0.97, \mathrm{p}=0.46)$, with a sharp decrease after age 30. An opposite pattern of patient distribution across age classes was observed for manifest HD, in which the telomere length in the HD patients under 50 years was lower than that of the oldest controls. Only a further slight decrease in telomere length with increasing age was noted (Table 2). After age 30 the differences between the mean LTL values of the three groups were always highly significant $(\mathrm{p}<0.0001)$.

After adjusting LTL for age, we analyzed the effect of CAG repeat number on LTL (Table 3). We noted an inverse relationship between mean LTL values and CAG repeats in the pre-manifest HD ( $\mathrm{p}=0.03$ ) but not in the manifest HD patients $(\mathrm{p}=0.66)$. This indicated that, in pre-manifest $\mathrm{HD}$, the CAG number in the pathological range contributes to LT attrition independent of age.

We then tried to test the hypothesis that in pre-HD LTL could be related to time to clinical diagnosis. As actual age at onset was not available, for each pre-HD subject, we calculated the estimated age at onset according to the formula of Langbehn et al. [20] at different probability values. A significant association between LTL and estimated years to HD onset (grouped into classes of 10 years) was observed (Table 4). Just as an example, the results related to the estimated years to HD onset at different probability values $(0.40,0.50,0.60,0.70)$ are shown. A common feature is that the mean LTL is below $0.70 \mathrm{~T} / \mathrm{S}$ in patients with an 
expected onset within 10 years or earlier, which then increases steadily up about 1.0 in patients with an expected onset after 30 years or more. As the probability increases, so, too, the expected age of onset, and the relationship weakens. According to the formula, the number of patients in each class varies as onset age changes according to the probability value. The relationship between LTL and estimated years to HD diagnosis, is better shown in fig. 2, where, as an example, the linear positive correlation between LTL and estimated years to HD diagnosis with $\mathrm{p}=60 \%$ in pre- manifest HD patients is reported $(\mathrm{y}=0.011 \mathrm{x}+0.60, \mathrm{p}<0.00001)$.

In the HD patients for whom the age of onset was known $(n=40)$, the disease duration was calculated as the difference between the reported age of onset and the age at blood sampling. No relationship was observed between disease duration for the HD patients and LTL adjusted for age ( $\mathrm{p}=0.77$ ) (Table 5), suggesting that no further major telomere attrition occurs after clinical HD onset.

\section{Discussion}

We investigated LTL of HD patients at different stages of disease (pre-manifest and manifest HD) and compared it with LTL of healthy controls. The mean LTL differed significantly between the three groups, with the highest mean values noted in the controls, intermediate values in the pre-manifest $\mathrm{HD}$, and the lowest values in the manifest HD patients. The difference suggests a definite relationship between telomere shortening and manifest HD development. The mean LTL values in the manifest HD patients were almost half those in the controls and slightly higher than the minimum length reported to be necessary to ensure human telomere protective stability in white blood cells [27]. Our finding is shared by the only other study that examined LTL in manifest HD patients [19], indicating that shortened LT might be a characteristic of HD patients. By direct comparison, the LTL of the HD patients was lower than that in patients with dementia [19] and $\mathrm{AD}$ [17], the other neurodegenerative disease in which LTL has been extensively investigated $[14,15]$. Of note is that telomere length in pre-manifest HD has never been examined before. In pre-manifest HD we found intermediate LTL values between the control and the manifest HD groups. This finding is consistent with similar observations in amnestic MCI, the prodromal stage of $\mathrm{AD}$, and seems to indicate a common 
model for neurodegenerative diseases in which initial telomere shortening is associated with initial stages of disease.

Given the well-known inverse relationship between LTL and age in the normal population, we examined it in the three groups. The most relevant result concerns the relationship LTL/age of pre-manifest HD subjects. It emerges (Table 2) that at an early age (below 30 years) LTL of pre-manifest HD and controls are not different, but, after age 30, LTL values in the pre-manifest HD are lower than those in controls after age 80 [17]. The majority of the youngest manifest HD patients (age over 30 years) were noted to have very short telomeres, and only a slight decrease was observed in the more advanced age classes.

The CAG repeat number was also associated with telomere shortening, but only in the pre-manifest HD patients, and was slightly less relevant than age.

The overall data seem to indicate that, in pre-manifest HD patients, leukocyte telomeres begin to shorten gradually and markedly after age 30 years, according to advancing age and CAG number, up to values typically observed in manifest HD patients. According to these findings, LT shortening seems to be a feature of HD development, from the pre-manifest stage to clinical diagnosis, but the actual meaning of this observation remains to be elucidated. An unresolved problem is whether shortening of leukocyte telomeres reflects a similar phenomenon at the neuronal level, since the absent/low level of cell turnover suggests that telomere shortening may not occur in the brain [10]. Studies on telomere length in brain tissue from AD patients, where LTL has been extensively investigated, have provided inconsistent results [2830]. On the other hand, LT shortening could be understood as part of HD physiopathology in which cell proliferation is activated by chronic systemic inflammation and oxidative stress. There is evidence that toxic HTT aggregates are accompanied by a neuroinflammatory state characterized by activation of the microglia in the brain of HD patients [31-33]. Microglia activation can be observed early in pre-manifest $\mathrm{HD}$, followed by the release of proinflammatory mediators. Elevated levels of proinflammatory mediators are also found in the blood of HD patients, including pre-manifest carriers, and many years before disease onset. Microglia activation also promotes the production of reactive oxygen species (ROS), and 
oxidative stress is known to accelerate telomere shortening [34-36]. In this context we may speculate that LT shortening is an indicator of the phenomena accompanying disease progression from the prodromal states (pre-manifest HD) to the full-blown HD.

The length of the CAG repeat is negatively correlated with age at disease onset, accounting for about 50-70\% of the variation in the age at onset [2]. On the basis of this relationship, some statistical models providing sufficiently accurate predictions have been proposed [20,37], although confounding factors might limit the usefulness of mathematical models. The identification of reliable biomarkers could greatly facilitate the prediction of disease onset and help in accurately tracking disease progression in pre-manifest HD. The above results allowed us to hypothesize that in pre-manifest HD, LTL could be a measure of time to clinical HD onset. To test this hypothesis, we examined the relationship between LTL and estimated time to clinical diagnosis calculated according to the formula of Langbehn et al. [20]. The relationship suggests that, in pre-manifest HD, with a probability well higher than $50 \%$, LTL $<0.70 \mathrm{~T} / \mathrm{S}$ could be associated with an estimated time to clinical onset of 10 years or less, $\mathrm{LTL}<0.80 \mathrm{~T} / \mathrm{S}$ with an estimated time to clinical onset of 20 years or less, and LTL $>0.80 \mathrm{~T} / \mathrm{S}$ with an estimated time to clinical onset of over 20 years. At over three decades before HD diagnosis, LTL values are indistinguishable from those expected for young, healthy subjects, a finding which is in line with the previous observation (Table 3) that, in very young patients, the LTL of pre-manifest HD was similar to that of the controls. The present preliminary results seem to indicate that LTL is a possible biomarker of HD progression, although it needs to be accurately validated by testing the relationship between LTL and the actual age at disease onset. Extending the analysis to a larger sample with a well-designed follow-up study would be useful to verify the actual relationships between LTL, age and time to disease onset. In addition, LTL measurement seems to possess some of the characteristics requisite for an ideal biomarker of HD progression [21]. It can be obtained by blood sampling and, in our experience, LTL is readily quantifiable and reproducible, although numerous studies provided evidence that some methodological conditions (for example DNA extraction method, or master mix used) can affect the telomere length measurement [38-40]. The final goal is to use LTL to implement and 
improve the prediction of age at disease onset provided by statistical model based on CAG repeat length. This result would be important for the development and evaluation of therapeutic interventions that slow or halt the progression of disease before symptoms begin.

\section{Acknowledgements}

This work was supported by grants 2016/2017 from Sapienza University of Rome.

We wish to thank A.I.C.H. Roma, HD patients and their relatives, and K.A. British for checking the manuscript style.

\section{Conflict of Interest}

The authors have no competing interests to declare. 


\section{References}

[1] R.H. Myers, Huntington's disease genetics, NeuroRx 1 (2004) 255-62.

[2] J.F. Gusella, M.E. MacDonald, J.M. Lee, Genetic modifiers of Huntington's disease. Mov. Disord. 29 (2014) 1359-65.

[3] C. Bettencourt, D. Hensman-Moss, M. Flower, S. Wiethoff, A. Brice, C. Goizet, et al., DNA repair pathways underlie a common genetic mechanism modulating onset in polyglutamine diseases, Ann. Neurol. 79 (2016) 983-90.

[4] J.M. Wong, K. Collins, Telomere maintenance and disease, Lancet 362 (2003) 983-8.

[5] E.H. Blackburn, C.W. Greider, J.W. Szostak, Telomeres and telomerase, the path from maize, Tetrahymena and yeast to human cancer and aging, Nat. Med. 12 (2006) 11331138.

[6] A. Ishaq, P.S. Hanson, C.M. Morris, G. Saretzki, Telomerase activity is downregulated early during human brain development, Genes 7 (2016) 27.

[7] R.M. Cawthon, K.R. Smith, E. O'Brien, A. Sivatchenko, R.A. Kerber, Association between telomere length in blood and mortality in people aged 60 years or older, Lancet 361 (2003) 393-5.

[8] M. Kimura, J.V. Hjelmborg, J.P. Gardner, L. Bathum, M. Brimacombe, X. Lu, et al., Telomere length and mortality, a study of leukocytes in elderly Danish twins, Am. J. Epidemiol. 167 (2008) 799-806.

[9] K. Lapham, M.N. Kvale, J. Lin, S. Connell, L.A. Croen, B.P. Dispensa, et al., Automated Assay of Telomere Length measurement and Informatics for 100,000 Subjects in the Genetic Epidemiology Research on Adult Health and Aging (GERA) Cohort, Genetics 200 (2015) 1061-72.

[10] K. Nakamura, K. Takubo, N. Izumiyama-Shimomura, M. Sawabe, T. Arai, H. Kishimoto, et al., Telomeric DNA length in cerebral gray and white matter is associated with longevity in individuals aged 70 years or older, Exp. Gerontol. 42 (2007) 944-50.

[11] J.L. Sanders, A.B. Newman, Telomere length in epidemiology, a biomarker of aging, age- related disease, both, or neither? Epidemiol. Rev. 35 (2013) 112-31.

[12] V. Codd, C.P. Nelson, E. Albrecht, M. Mangino, J. Deelen, J.L. Buxton, J.J. Hottenga, et al., Identification of seven loci affecting mean telomere length and their association with disease, Nat. Genet. 45 (2013) 422-7.

[13] J.H. Barrett, M.M. Iles, A.M. Dunning, K.A. Pooley, Telomere length and common disease, study design and analytical challenges, Hum. Genet. 34 (2015) 679-89.

[14] Z. Cai, J. Yan, A. Ratka, Telomere shortening and Alzheimer's disease, Neuromolecular Med. 15 (2013) 25-48. 
[15] V. Boccardi, L. Pelini, S. Ercolani, C. Ruggiero, P. Mecocci, From cellular senescence to Alzheimer's disease The role of telomere shortening, Ageing Res. Rev. 22 (2015) 1 8.

[16] D.A. Forero, Y. González-Giraldo, C. López-Quintero, L.J. Castro-Vega, G.E. Barreto, G. Perry, Meta-analysis of Telomere Length in Alzheimer's Disease, J. Gerontol. A Biol. Sci. Med. Sci. 71 (2016) 1069-73.

[17] D. Scarabino, E. Broggio, G. Gambina, R.M. Corbo, Leukocyte telomere length in mild cognitive impairment and Alzheimer's disease patients, Exp. Gerontol. 98 (2017) 143-147.

[18] D.A. Forero, Y. González-Giraldo, C. López-Quintero, L.J. Castro-Vega, G.E. Barreto, G. Perry, Telomere length in Parkinson's disease: A meta-analysis, Exp. Gerontol. 75 (2016) 53-5.

[19] L.N. Kota, S. Bharath, M. Purushottam, N.S. Moily, P.T. Sivakumar, M. Varghese, et al., Reduced telomere length in neurodegenerative disorders may suggest shared biology, J. Neuropsychiatry Clin. Neurosci. 27 (2015) e92-6.

[20] D.R. Langbehn, R.R. Brinkman, D. Falush, J.S. Paulsen, M.R. Hayden, International Huntington's Disease Collaborative Group, A new model for prediction of the age of onset and penetrance for Huntington's disease based on CAG length, Clin. Genet. 65 (2004) 267-77.

[21] D.W. Weir, A. Sturrock, B.R. Leavitt, Development of biomarkers for Huntington's disease, Lancet Neurol. 10 (2011) 573-90.

[22] C.A. Ross, E.H. Aylward, E.J.Wild, D.R. Langbehn, J.D. Long, J.H. Warner, R.I. Scahill, B.R. Leavitt, J.C. Stout, J.S. Paulsen, R. Reilmann, P.G. Unschuld, A. Wexler, R.L. Margolis, S.J. Tabrizi. Huntington disease: natural history, biomarkers and prospects for therapeutics. Nat Rev Neurol. 10 (2014) 204-16.

[23] S.A. Miller, D.D. Dykes, H.F. Polesky, A simple salting out procedure for extracting DNA from human nucleated cells, Nucleic Acids Res. 6 (1988) 1215.

[24] J.P. Warner, L.H. Barron, D.J. Brock, A new polymerase chain reaction (PCR) assay for the trinucleotide repeat that is unstable and expanded on Huntington's disease chromosomes, Mol. Cell Probes 7 (1993) 235-9.

[25] J.P. Warner, L.H. Barron, D. Goudie, K. Kelly, D. Dow, D.R. Fitzpatrick, D.J. Brock, A general method for the detection of large CAG repeat expansions by fluorescent PCR, J. Med. Genet. 33 (1996) 1022-6.

[26] R.M. Cawthon, Telomere measurement by quantitative PCR, Nucleic Acids Res. 30 (2002) e47.

[27] E.H. Blackburn, E.S. Epel, J. Lin, Human telomere biology: A contributory and interactive factor in aging, disease risks, and protection, Science 350 (2015) 1193-8. 
[28] S. Franco, M.A. Blasco, S.L. Siedlak, P.L. Harris, P.I. Moreira, G. Perry, M.A. Smith, Telomeres and telomerase in Alzheimer's disease, epiphenomena or a new focus for therapeutic strategy ? Alzheimers Dement. 2 (2006) 164-8.

[29] J.N. Lukens, V. Van Deerlin, C.M. Clark, S.X. Xie, F.B. Johnson, Comparisons of telomere lengths in peripheral blood and cerebellum in Alzheimer's disease, Alzheimers Dement. 5 (2009) 463-9.

[30] P. Thomas, N.J. O' Callaghan, M. Fenech, Telomere length in white blood cells, buccal cells and brain tissue and its variation with ageing and Alzheimer's disease, Mech. Ageing Dev. 129 (2008) 183-90.

[31] L.F. Clark, T. Kodadek, The Immune System and Neuroinflammation as Potential Sources of Blood-Based Biomarkers for Alzheimer's Disease, Parkinson's Disease, and Huntington's Disease, ACS Chem. Neurosci. 7 (2016) 520-527.

[32] R. Andre, L. Carty, S.J. Tabrizi, Disruption of immune cell function by mutant huntingtin in Huntington's disease pathogenesis, Curr. Opin. Pharmacol. 26 (2016) 338.

[33] E. Mina, W. van Roon-Mom, K. Hettne, E. van Zwet, J. Goeman, C. Neri, et al., Common disease signatures from gene expression analysis in Huntington's disease human blood and brain, Orphanet J. Rare Dis. 11 (2016) 97.

[34] E. Eitan, E.R. Hutchison, M.P. Mattson, Telomere shortening in neurological disorders: an abundance of unanswered questions, Trends Neurosci. 37 (2014) 256-63.

[35] T. von Zglinicki, Oxidative stress shortens telomeres, Trends Biochem. Sci. 27 (2002) 339-344.

[36] M. Ragno, L. Pianese, M. Pinelli, S. Silvestri, G. Cacchiò, F. Di Marzio, et al., Shorter telomeres in patients with cerebral autosomal dominant arteriopathy and leukoencephalopathy (CADASIL), Neurogenetics 12 (2011) 337-43.

[37] D.R. Langbehn, M. Hayden, J. S. Paulsen, CAG-Repeat Length and the Age of Onset in Huntington Disease (HD): A Review and Validation Study of Statistical Approaches. Am. J. Med. Genet. B Neuropsychiatr. Genet. 2 (2010) 397-408.

[38] G. Aubert, M. Hills, P.M. Lansdorp, Telomere length measurement-caveats and a critical assessment of the available technologies and tools. Mutat. Res. 730 (2012) 5967.

[39] J. Denham, F.Z. Marques, F.J. Charchar, Leukocyte telomere length variation due to DNA extraction method, BMC Research Notes 7 (2014) 877.

[40] K. M. Jiménez, D. A. Forero, Effect of master mixes on the measurement of telomere length by qPCR. Mol. Biol. Rep. 45 (2018) 633-638. 
Table 1 Demographic and clinical characteristics of the three groups

\begin{tabular}{llll}
\hline & \multicolumn{1}{c}{$\begin{array}{c}\text { Controls } \\
\mathrm{n}=76\end{array}$} & $\begin{array}{c}\text { Pre-manifest HD } \\
\text { patients } \\
\mathrm{n}=38\end{array}$ & \multicolumn{1}{c}{$\begin{array}{c}\text { Manifest HD patients } \\
\mathrm{n}=62\end{array}$} \\
\hline Age (yrs) & $\begin{array}{l}49.8 \pm 15.1 \\
(\text { range, 19-74) }\end{array}$ & $\begin{array}{l}35.9 \pm 11.7 \\
(\text { range, 19-62) }\end{array}$ & $\begin{array}{l}55.3 \pm 11.7 \\
\text { (range, 20-77) }\end{array}$ \\
Sex (males, \%) & 32.4 & 42.1 & 50.8 \\
CAG (median and range) & $/$ & $43(40-52)$ & $42(40-69)$ \\
Age of onset (yrs) & $/$ & $/$ & $\begin{array}{l}51.0 \pm 12.8 \\
(\text { range, 23-76) }\end{array}$ \\
& & &
\end{tabular}


Table 2 LTL distribution (T/S ratio) in controls, pre-manifest HD, and HD patients by age class in years (median, first and third quartiles).

In brackets the number of subjects

\begin{tabular}{llll}
\hline $\begin{array}{l}\text { Age class } \\
(\text { yrs. })\end{array}$ & \multicolumn{1}{c}{ Controls } & Pre-manifest HD patients & \multicolumn{1}{c}{ HD patients } \\
\hline Total sample & $0.92,0.86-0.98(76)$ & $0.76,0.69-0.88(38)$ & $0.56,0.53-0.62(62)$ \\
$20-29$ & $0.97,0.94-0.98(10)$ & $0.91,0.8-1.0(14)$ & $0.63^{\mathrm{a}}(1)$ \\
$30-39$ & $0.96,0.9-1.03(11)$ & $0.71,0.68-0.80(10)$ & $0.63,0.6-0.8^{\mathrm{a}}(3)$ \\
$40-49$ & $0.96,0.86-1.0(14)$ & $0.68,0.56-0.79(10)$ & $0.61,0.55-0.66(15)$ \\
$50-59$ & $0.91,0.88-1.0(14)$ & $0.61(2)$ & $0.55,0.5-0.6(19)$ \\
$60-69$ & $0.91,0.84-0.96(21)$ & $0.77(2)$ & $0.55,0.53-0.56(16)$ \\
$70-79$ & $0.81,0.76-0.87(6)$ & & $0.56,0.51-0.61(8)$ \\
P value & 0.005 & 0.004 & 0.012
\end{tabular}

${ }^{\mathrm{a}}$ These samples were pooled for the analysis 
Table 3 Effect of CAG repeat number on LTL (T/S).

In brackets the number of subjects

\begin{tabular}{lll}
\hline CAG repeats & Pre-manifest HD & \multicolumn{1}{c}{ HD patients } \\
& & \\
\hline $40-41$ & $0.86 \pm 0.13(13)$ & $0.57 \pm 0.05(16)$ \\
$42-43$ & $0.77 \pm 0.13(6)$ & $0.56 \pm 0.05(26)$ \\
$44-45$ & $0.74 \pm 0.11(13)$ & $0.58 \pm 0.08(14)$ \\
$\geq 46$ & $0.70 \pm 0.14(6)$ & $0.63 \pm 0.12(6)$ \\
P value & 0.03 & 0.66
\end{tabular}


Table 4 Relationship between mean LTL (T/S) of pre-manifest HD mutation carriers and estimated years to HD onset (grouped into classes of 10 years) at different probability values.

\begin{tabular}{lllll}
\hline $\begin{array}{l}\text { Estimated years } \\
\text { to HD onset }\end{array}$ & \multicolumn{1}{c}{$\mathrm{P}=0.40$} & \multicolumn{1}{c}{$\mathrm{P}=0.50$} & \multicolumn{1}{c}{$\mathrm{P}=0.60$} & $\mathrm{P}=0.70$ \\
\hline$\leq 10$ & $0.70 \pm 0.14(17)$ & $0.69 \pm 0.14(16)$ & $0.69 \pm 0.15(14)$ & $0.66 \pm 0.11(9)$ \\
$11-20$ & $0.80 \pm 0.12(13)$ & $0.77 \pm 0.12(10)$ & $0.76 \pm 0.12(11)$ & $0.74 \pm 0.12(14)$ \\
$21-30$ & $0.89 \pm 0.13(6)$ & $0.89 \pm 0.13(9)$ & $0.85 \pm 0.12(9)$ & $0.86 \pm 0.12(9)$ \\
$>30$ & $1.04 \pm 0.08(2)$ & $0.99 \pm 0.08(3)$ & $1.02 \pm 0.08(4)$ & $0.94 \pm 0.08(6)$ \\
P value & 0.002 & 0.0006 & 0.0004 & 0.002
\end{tabular}


Table 5 Relationship between disease duration of HD patients and LTL (T/S).

\begin{tabular}{ll}
\hline $\begin{array}{l}\text { Years since HD } \\
\text { onset (duration) }\end{array}$ & LTL (T/S) \\
\hline $0-1$ & $0.59 \pm 0.10(10)$ \\
$2-3$ & $0.55 \pm 0.07(10)$ \\
$4-5$ & $0.58 \pm 0.10(8)$ \\
$6-10$ & $0.58 \pm 0.05(8)$ \\
$>10$ & $0.61 \pm 0.07(4)$ \\
P value & 0.77
\end{tabular}




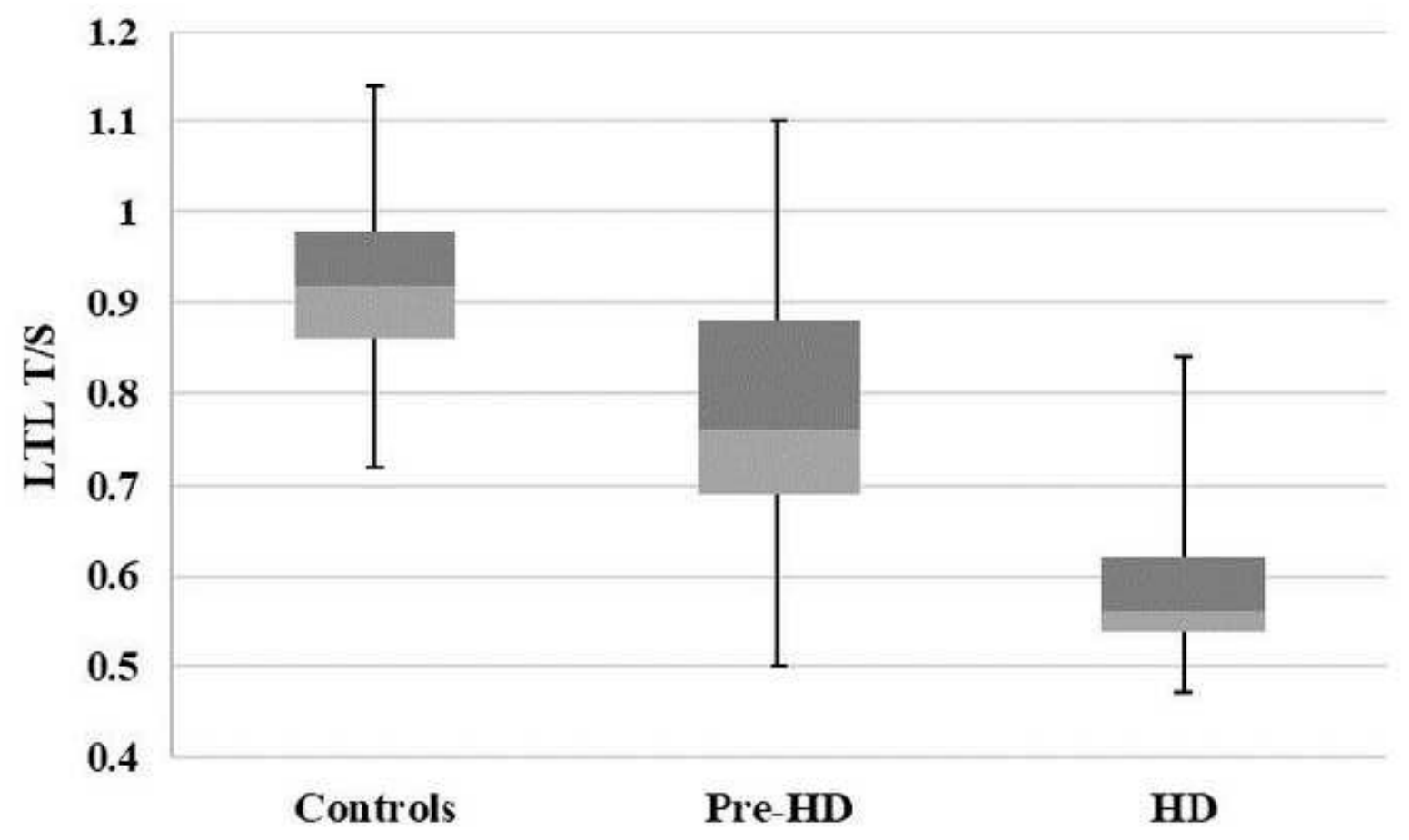

Fig. 1 Box Plot Showing the distribution of LTL (T/S ratio) in controls, pre-manifest HD, and HD patients. Controls: median $=0.92$, first quartile $\left(\mathrm{q}^{1}\right)=0.86$, third quartile $\left(\mathrm{q}^{3}\right): 0.62$; premanifest HD: median $=0.76, \mathrm{q}^{1}=0.69, \mathrm{q}^{3}=0.88 ;$ HD patients: median $=0.56, \mathrm{q}^{1}=0.53, \mathrm{q}^{3}$ : 0.62 . 


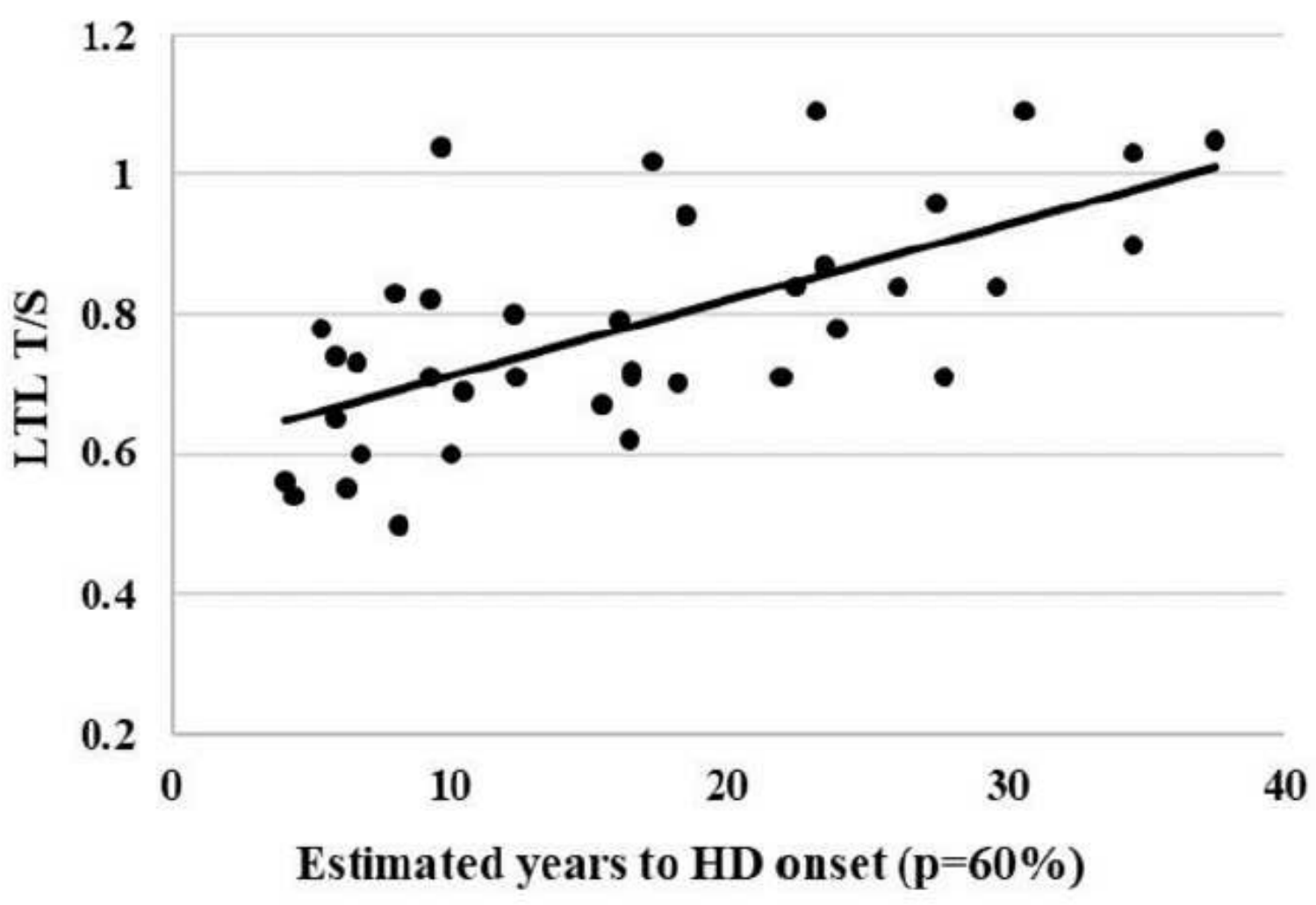

Fig. 2 Relationship between LTL (T/S) and estimated years to HD diagnosis ( $\mathrm{p}=60 \%$ ) in pre-manifest HD patients. 